\title{
Sleep and duodenal motor activity in patients with severe non-ulcer dyspepsia
}

\author{
D David, H Mertz, L Fefer, B Sytnik, H Raeen, N Niazi, A Kodner, E A Mayer
}

\begin{abstract}
The prevalence of sleep disturbances was studied in patients with severe non-ulcer dyspepsia. It was also considered if the change in sleep pattern was associated with changes in the rhythmic fasting motor activity of the gastrointestinal tract, and if motor events correlate with the patient's symptoms. Motor activity in the duodenum was monitored over a 24 hour period under freely ambulatory conditions in $\mathbf{1 0}$ healthy controls and in $\mathbf{1 0}$ patients with severe non-ulcer dyspepsia using a transnasally placed catheter with six solid state pressure transducers connected to a digital data logging device. Symptoms and sleep disturbance were assessed by questionnaire and diary. Based on their symptoms, the patients were separated into two groups: those with dyspepsia symptoms only (non-ulcer
\end{abstract} dyspepsia; $n=5$ ) and those with dyspepsia and additional functional symptoms thought to arise from the lower gastrointestinal tract (non-ulcer dyspepsia+irritable bowel syndrome; $n=5$ ). When compared with either the control or the non-ulcer dyspepsia +irritable bowel syndrome group, non-ulcer dyspepsia patients had a considerably decreased number of migrating motor complexes during the nocturnal period $(0.7 v 4.6)$, a decreased percentage of nocturnal phase I $(5 \cdot 2 \% v 78 \cdot 0 \%)$, and an increased percentage of the nocturnal period in phase II $(94 \% v 15 \cdot 4 \%)$. Patients with non-ulcer dyspepsia+irritable bowel syndrome were not different from normal controls. Four of the non-ulcer dyspepsia patients and all of the non-ulcer dyspepsia +irritable bowel syndrome patients reported difficulties with sleep. Clusters of high amplitude tonic and phasic activity, not accompanied by subjective reports of discomfort were noted in several patients in both groups during the study. In eight of 10 patients, abdominal pain was reported during normal motor activity, while in one patient, pain correlated with phase III of the migrating motor complex. In contrast with previous reports in patients with irritable bowel syndrome, our findings suggest an abnormality of diurnal rhythmicity - shown in changed sleep and changed rhythmic duodenal motor activity - in patients with chronic abdominal pain thought to arise from the upper gastrointestinal tract.

(Gut 1994; 35: 916-925)
The syndrome of non-ulcer dyspepsia comprises a heterogeneous group of functional disorders assumed to arise from the upper gut, which share common symptoms: upper abdominal pain or discomfort, bloating, gas, nausea, and early satiety. ${ }^{1-3}$ Although $50 \%$ of these patients show evidence for delayed gastric emptying or postprandial antral hypomotility, or both ${ }^{4-6}$ there is little evidence for a primary aetiological role of the motor abnormalities in most non-ulcer dyspepsia patients. ${ }^{7}{ }^{8} \mathrm{~A}$ considerable number of patients with non-ulcer dyspepsia complain also of symptoms thought to arise from the lower gastrointestinal tract consistent with a diagnosis of the irritable bowel syndrome. ${ }^{9}$ Recent evidence suggests that many non-ulcer dyspepsia patients have a lowered threshold for conscious perceptions of gastric balloon distension, consistent with gastric hyperalgesia. ${ }^{10} 11$

Absence of gastrointestinal symptoms during sleep has generally been taken as a clinical indication for the presence of 'functional' $v$ 'organic' disease. ${ }^{12}$ Many patients, however, with functional bowel disorders including nonulcer dyspepsia will give a history of difficulties with sleep, including frequent awakenings with or without pain, and non-regenerative sleep. ${ }^{13} 14$ Non-regenerative sleep associated with a specific pattern on the sleep electroencephalogram (the alpha wave intrusion, or 'alpha abnormality') has been described in the fibromyalgia syndrome, ${ }^{15-18}$ a chronic somatic pain syndrome. It has been suggested that the 'alpha anomaly' may be a genetically determined trait, which can occur in asymptomatic subjects but may only manifest itself clinically under situations of increased stress. ${ }^{17}$ Fibromyalgia syndrome occurs in up to $60 \%$ of patients with functional bowel disorders, ${ }^{19-21}$ and close to $50 \%$ of patients with a diagnosis of fibromyalgia syndrome complain of nausea, abdominal pain, and bloating, which are symptoms characteristic of non-ulcer dyspepsia. ${ }^{17}$ Similar to patients with fibromyalgia syndrome, ${ }^{17-22}$ a subset of patients with different functional bowel disorders respond to low doses of tricyclic antidepressants. ${ }^{23}$ The effect of this type of treatment, which is independent of mood raising properties is possibly related to the effect of returning the sleep pattern to normal. ${ }^{24} 25$ Recent studies using prolonged ambulatory monitoring of duodenal motor activity in patients with irritable bowel syndrome have not shown a change in nocturnal motor patterns. ${ }^{26}$ In addition, no abnormalities of non-rapid eye movement (non-REM) sleep have been seen in irritable bowel syndrome patients. $^{27}$ 
In this study, we sought to answer three questions: (1) What is the prevalence of subjective sleep disturbances in non-ulcer dyspepsia patients? (2) Is the sleep disturbance associated with a change in rhythmicity of nocturnal duodenal motor activity? (3) Are the patient's typical symptoms associated with specific duodenal motor events during freely ambulatory conditions.

\section{Methods}

\section{SUBJECTS}

\section{Normal volunteers}

Ten healthy male subjects between the ages of 28 and 60 (mean age $45 \cdot 5$ ) were recruited by advertisement. Previous studies in humans have failed to show significant sex differences in fasting motor activity, ${ }^{27}$ and animal studies have not shown a consistent effect of female sex hormones on small intestinal myoelectric activity. $^{28}$ None of the subjects had any evidence (by history, physical examination or upper gastrointestinal endoscopy) for an acute or chronic illness, or for a chronic pain problem. In particular, sleep problems, and symptoms consistent with non-ulcer dyspepsia, irritable bowel syndrome or fibromyalgia syndrome were ruled out using the same questionnaires used for assessment of bowel symptoms ${ }^{29}$ and sleep disturbance as in the non-ulcer dyspepsia patients. The study was approved by the Veterans Administration Wadsworth research and human studies committees, and informed consent was obtained from each subject.

\section{NON-ULCER DYSPEPSIA PATIENTS}

All patients with non-ulcer dyspepsia were tertiary referrals for special diagnostic evaluation to the UCLA Center for Functional Bowel Disorders and Abdominal Pain. All patients had seen more than one subspecialist for their problem before referral to UCLA. A diagnosis of non-ulcer dyspepsia, and of non-ulcer dyspepsia +irritable bowel syndrome was made using a modified Talley bowel symptom questionnaire $^{29}$ and a complete evaluation by one of three gastroenterologists who specialised in functional bowel disorders. A diagnosis of non-ulcer dyspepsia was made when a patient gave a history of at least three months of abdominal pain or discomfort, and a sensation of bloating or fullness thought to arise from the upper gastrointestinal tract, including nausea and early satiety. ${ }^{30}$ Patients who had had previous gastric surgery or with a history of peptic ulcer disease were excluded. A diagnosis of irritable bowel syndrome was made according to the Rome criteria, ${ }^{31}$ and the number of symptoms was quantified using the Manning criteria. ${ }^{32}$

\section{Non-ulcer dyspepsia patients for manometric protocol}

Table I shows a summary of the clinical characteristics of the 10 patients who had ambulatory manometry. All patients were women (28-60 years of age; mean age 40 years) with longstanding disease (2-20 year duration of symptoms; mean duration nine years). Three patients were postmenopausal, one patient had had bilateral oophorectomy. Patients were recruited consecutively from the UCLA Functional Bowel Clinic for this study, and no patient who was offered the 24 hour manometry refused to have the test. Five patients only complained about symptoms thought to arise from the upper gastrointestinal tract (non-ulcer dyspepsia), whereas five patients had additional symptoms consistent with a diagnosis of irritable bowel syndrome (non-ulcer dyspepsia+irritable bowel syndrome. ${ }^{31} 32$ Non-ulcer dyspepsia + irritable bowel syndrome patients had at least $4 / 6$ positive Manning criteria, while non-ulcer dyspepsia patients had at most $2 / 6$ positive criteria (Table I). In the non-ulcer dyspepsia group, two patients were postmenopausal while three had menstrual cycles. Two patients reported exacerbation of baseline symptoms synchronous with the menstrual cycle while four did not report such a temporal association.

All patients had had an abdominal ultrasound examination and an oesophagogastroduodenoscopy to rule out organic disease within the last six months. Gastro-oesophageal reflux disease was ruled out in all subjects by previous 24 hour monitoring of oesophageal pH. Gastric emptying studies using a technetium-99M $\left({ }^{99 m} \mathrm{Tc}\right)$ labelled tuna salad sandwich showed mildly delayed gastric emptying in five patients (three in non-ulcer dyspepsia, two in non-ulcer dyspepsia + irritable bowel syndrome). Fifty per cent of

TABLE I Clinical characteristics of the 10 patients who had ambulatory manometry

\begin{tabular}{|c|c|c|c|c|c|c|c|}
\hline Age & Sex & $\begin{array}{l}\text { Symptom } \\
\text { duration }\end{array}$ & $\begin{array}{l}\text { Abdominal } \\
\text { pain }\end{array}$ & Nausea & Bloating & $\begin{array}{l}\text { Sleep } \\
\text { disturbance }\end{array}$ & $\begin{array}{l}\text { IBS } \\
\text { symptoms }\end{array}$ \\
\hline 43 & $\mathbf{F}$ & 24 Months & + & + & + & + & 0 \\
\hline 57 & $\mathbf{F}$ & 15 Years & + & + & + & + & + \\
\hline 34 & $\mathrm{~F}$ & 10 Years & + & + & + & + & 0 \\
\hline 37 & $\mathrm{~F}$ & 9 Years & + & + & + & + & + \\
\hline 30 & $\mathbf{F}$ & 28 Months & + & + & + & + & + \\
\hline 32 & $\mathbf{F}$ & 7 Years & + & + & + & 0 & 0 \\
\hline 28 & $\mathbf{F}$ & 4 Years & + & + & + & + & + \\
\hline 52 & $\mathbf{F}$ & 6 Years & + & + & + & + & 0 \\
\hline 60 & $\mathbf{F}$ & 20 Years & + & + & + & + & 0 \\
\hline 34 & F & 2 Years & + & + & + & + & + \\
\hline
\end{tabular}

* Indicates at least 1 of the following: difficulty falling asleep, awakening during the night, non-restorative sleep; $\dagger+$ indicates at least $4 / 6$ of the following: changed bowel habit with pain onset, pain relief after defecation, sensation of incomplete evacuation rectal mucus, urgency, straining; 0 indicates equal or less than 2 of the above criteria; $\ddagger+$ indicates nausea was present several times a week. IBS = irritable bowel syndrome. 
patients had a psychiatric diagnosis (depression, anxiety or somatisation disorder), and one third gave a history of sexual abuse. The prevalence of psychiatric diagnoses and psychosocial factors was not different from non-ulcer dyspepsia patients seen at UCLA with comparable symptom severity. Eighty per cent of patients gave a family history of functional bowel disorders. None of the 10 patients undergoing the manometric study were on narcotic, sleep or psychotropic drugs during the time of the study. Patients were asked to stop all drugs 48 hours before the beginning of the study. None of the patients were studied during their menstrual period.

\section{ASSESSMENT OF SLEEP DISTURBANCE}

The prevalence of subjective complaints suggestive of a chronic sleep disturbance was assessed in the 10 normal volunteers and in the 10 patients undergoing this study protocol (manometric protocol). In addition, sleep disturbance was assessed in 65 non-ulcer dyspepsia patients seen consecutively over a three month period at the UCLA Functional Bowel Center (40 female, 25 male; mean age 50 ; range 26-83), and in 43 control subjects (34 female; 9 male; mean age 36 ; range 22-71). Control subjects were recruited from the clerical staff of the department of medicine. Control subjects who complained of chronic abdominal symptoms or who had consulted a doctor for problems related to the gastrointestinal tract during the past year, were not included in the study.

A diagnosis of a sleep disturbance was made based on the response of the patient to the following questions regarding their sleep during the past year: (a) difficulty falling asleep; (b) frequent awakening; (c) awakening in the morning and feeling tired, or not rested (non-regenerative sleep); (d) worsening of sleep problems during worsening of bowel problems. Frequent awakenings were subdivided into episodes resulting from abdominal discomfort and those without symptoms. The subjects undergoing the manometric evaluation were given the questionnaire before the study.

The influence of the test situation on all subjects was evaluated by questionnaire using visual analogue scales after tube removal. Subjects were asked to rate the discomfort experienced by the manometry tube from mild (1) to severe (10). In addition, they were asked to compare the quality of sleep during the study with their usual sleep (1, same as usual; 5, somewhat worse; 10, considerably worse).

\section{MANOMETRIC RECORDINGS}

Antroduodenal motor activity was monitored with a highly elastic catheter containing six solid state pressure transducers (Koenigsberg Instruments, Pasadena, CA). The portion of the catheter located in the stomach and duodenum had a diameter of $4.65 \mathrm{~mm}$, whereas the more proximal portion of the catheter had a diameter of $2.8 \mathrm{~mm}$. Sensors were calibrated before placement using a manometric calibration system for solid state pressure catheters (Synectics, Irving, TX).

After an overnight fast, the catheter was placed transnasally into the stomach. Subsequently, the patient was placed in the left lateral decubitus position and the tip of the catheter was moved with biopsy forceps placed through a paediatric duodenoscope into the third portion of the duodenum. While the tip was held in place with the biopsy forceps, the endoscope was gradually withdrawn into the stomach. If the catheter moved during withdrawal of the endoscope, it was advanced again by grabbing it on one of four sites where a suture was attached to the catheter. For the endoscopic procedure the subject's throat was sprayed with $2 \%$ xylocaine and a maximal dose of $2 \mathrm{mg}$ of midazolam (Versed; Roche) was given intravenously for sedation. The amount of midazolam in $\mathrm{mg} / \mathrm{kg}$ body weight given to patients and normal subjects did not differ. The three proximal recording sites (spaced 1 $\mathrm{cm}$ apart) were placed under direct vision into the gastric antrum, whereas the three distal recording sites (spaced $10 \mathrm{~cm}$ apart) were placed into the duodenum. The distance between the most distal antral and the most proximal duodenal recording site was $10 \mathrm{~cm}$. The location of the catheter was verified fluoroscopically after placement and just before withdrawing the catheter at the end of the study. Based on comparison of these two radiographs, there was no significant change in the location of the sensors within the stomach, even though small transient movements of the tube during the study are possible, based on the motility records. The catheter was connected to a digital data logging device (MicroDigitrapper, version 93-6600, Synectics Corporation, Irving, TX), and the patient was instructed to push one of four event buttons to indicate symptoms, time of food intake, and sleep. After withdrawal of the catheter, the data were transferred from the logging device to a PC computer and data analysed using a previously validated analysis software. ${ }^{33}$

\section{STUDY PROTOCOL}

Recording of duodenal motor activity was started three hours after completion of catheter placement. Normal control subjects were freely ambulatory but were asked to stay on the premises of the VA campus, and slept in the clinical study centre of the hospital. They ate two standard meals and were asked to refrain from any additional meals, snacks or caloric drinks. The standard meals were eaten at lunch time (12 am; $400 \mathrm{kcal})$ and dinner (6 pm; $570 \mathrm{kcal})$.

Patients were freely ambulatory. Two slept in the clinical study centre, two in a hotel, and six at home. They were asked to eat only two meals of their own choice (at 12 am and $6 \mathrm{pm}$ ) during the time of the study, and to refrain from snacks, or caloric drinks. 
TABLE II Type and prevalence of sleep disturbances

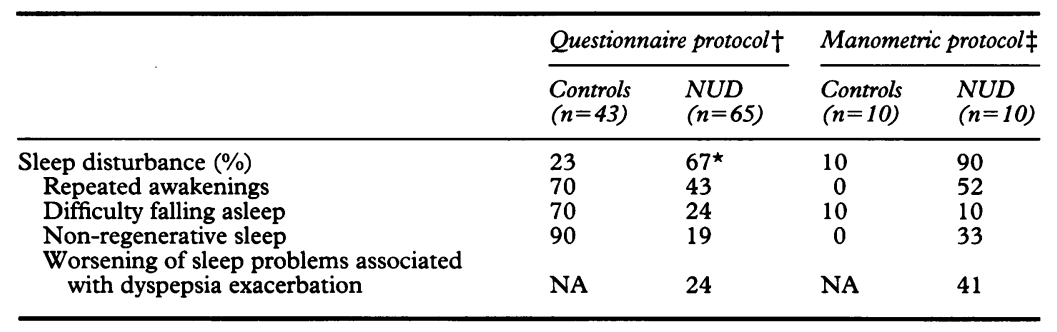

$\star(p<0.05) ; \dagger$ data from symptom questionnaire given to 43 asymptomatic controls and 65 consecutive clinic patients with NUD; $¥$ data from participants (controls and NUD patients) in the manometric study. As most subjects reported more than one symptom, percentages do not add up to $100 \%$. NUD = non-ulcer dyspepsia; NA=not applicable.

\section{DATA ANALYSIS}

Records of duodenal motor activity were analysed both visually (switching from fasting to postprandial, identification and quantification of duration of migrating motor complex phases, identification of a change in motor patterns) and using a previously validated software (to calculate motility index). ${ }^{33}$ Because of the inconsistency of antral records (probably because of the transient movements of the tube during the study, in particular during food intake), antral motor activity was not further analysed. Pressure changes $<5 \mathrm{~mm} \mathrm{Hg}$ and obvious artifacts (such as simultaneous pressure changes occurring at all six sensors) were excluded from analysis. Each study was divided into a diurnal and a nocturnal period. The nocturnal period was defined as the period of recording between the recorded time of going to bed (as shown by the patient) and the time of getting up in the morning (as shown by the patient and apparent from a clear cut increase in phase II motor activity). Awake periods occurring during the night were included in the nocturnal period.

The remainder of the study was identified as the diurnal period. During the diurnal period, the times at which subjects ate (postprandial period) were determined $(14.5 \%$ of diurnal time). The end of the postprandial phase was defined at the point at which phase III of the migrating motor complex first reappeared after a meal. The evening postprandial periods were similar between controls and patients. The postprandial period was not further analysed in this study. The remainder of the diurnal recording, designated as fasting and the entire nocturnal period, were analysed for motor activity characteristic of phase I (motor quiescence), phase II (irregular contractile activity), or phase III (regular contractile activity at a frequency of $11-13 \mathrm{~min}^{-1}$ ), of the migrating motor complex. ${ }^{34} 35$

The duration and the percentage of diurnal (total diurnal minus postprandial) and nocturnal recording time occupied by phase I, phase II, and phase III of the migrating motor complex were determined. Values obtained from each of the three duodenal recording sites were averaged. The migrating motor complex cycle length, defined as the interval between two successive phase III activity fronts during which the subject did not eat or drink, was noted.

In addition, the duration and rate of aboral propagation of the migrating motor complex phase III was determined as previously described. ${ }^{34}$ The record was analysed for the presence of a change in motor patterns such as discrete clustered contractions, or tonic contractions, ${ }^{35} 36$ and for the possible correlation of motor activity with subjective symptoms recorded by the patient during the study period. A motor event was considered as correlated with symptoms if it occurred within five minutes before or after the symptom report.

\section{STATISTICAL ANALYSIS}

Data are expressed throughout the study as mean (SEM). Statistical significance was assessed using Student's $t$ test for unpaired data.

\section{Results}

QUESTIONNAIRE PROTOCOL

Table II summarises the type and prevalence of sleep disturbances of the 43 control subjects and the 65 non-ulcer dyspepsia subjects. Both the complaint of general sleep disturbances and symptoms suggestive of non-regenerative sleep (waking up repeatedly, morning awakening without feeling rested) were significantly more common in non-ulcer dyspepsia patients $(67 \%)$ than in control subjects $(23 \%)$ $(\mathrm{p}<0.05)$. When only women were compared, $75 \%$ of non-ulcer dyspepsia patients and $29 \%$ of controls complained of difficulty with sleep $(\mathrm{p}<0.05)$.

Of the 28 non-ulcer dyspepsia patients who reported repeated nocturnal awakenings, $65 \%$ blamed the awakening on their abdominal symptoms, $15 \%$ did not know the reason why they awoke, but experienced pain after awakening, and $20 \%$ stated they awoke because of urinary urgency.

MANOMETRIC PROTOCOL

Table II summarises the corresponding values for subjective sleep disturbances for the 10 normal volunteers and the 10 non-ulcer dyspepsia patients undergoing the manometric study. Ninety per cent of non-ulcer dyspepsia patients reported difficulties with sleep, with the complaints of frequent awakenings and non-regenerative sleep being most common. The type of gastrointestinal symptoms (symptoms thought to arise from upper or lower gastrointestinal tract) had no influence

TABLE III Phases of the migrating motor complex (MMC) (mean (SEM))

\begin{tabular}{|c|c|c|c|}
\hline & $\begin{array}{l}\text { Controls } \\
(n=10)\end{array}$ & $\begin{array}{l}N U D \\
(n=5)\end{array}$ & $\begin{array}{l}\text { NUD +IBS } \\
(n=5)\end{array}$ \\
\hline \multicolumn{4}{|c|}{ Diurnal proportion of MMC phase: } \\
\hline Phase $1(\%)$ & $0 \cdot 8(0 \cdot 1)$ & $3.9(1.0)^{\star}$ & $1 \cdot 3(0.6)$ \\
\hline Phase $2(\%)$ & $98 \cdot 4(1 \cdot 2)$ & $94 \cdot 4(9 \cdot 7)$ & $97 \cdot 7(1 \cdot 7)$ \\
\hline Phase $3(\%)$ & $0.8(0.2)$ & $1.7(0.4)$ & $1.0(0.5)$ \\
\hline \multicolumn{4}{|c|}{ Nocturnal proportion of MMC phase: } \\
\hline Phase $1(\%)$ & $78 \cdot 3(6 \cdot 0)$ & $5 \cdot 2(1.9)^{\star \star}$ & $79 \cdot 1(6 \cdot 7)$ \\
\hline Phase $2(\%)$ & $15 \cdot 4(2 \cdot 4)$ & $93.8(2.9)^{\star \star}$ & $17 \cdot 6(7 \cdot 4)$ \\
\hline Phase $3(\%)$ & $6 \cdot 3(1 \cdot 0)$ & $1 \cdot 0(0 \cdot 3)^{\star}$ & $3 \cdot 3(1 \cdot 1)$ \\
\hline
\end{tabular}

${ }^{\star} \mathrm{p}<0.05 v$ controls; ${ }^{\star \star} \mathrm{p}<0.005 v$ controls. $\mathrm{MMC}=$ migrating motor complex; other abbreviations as in Tables I and II. 
A

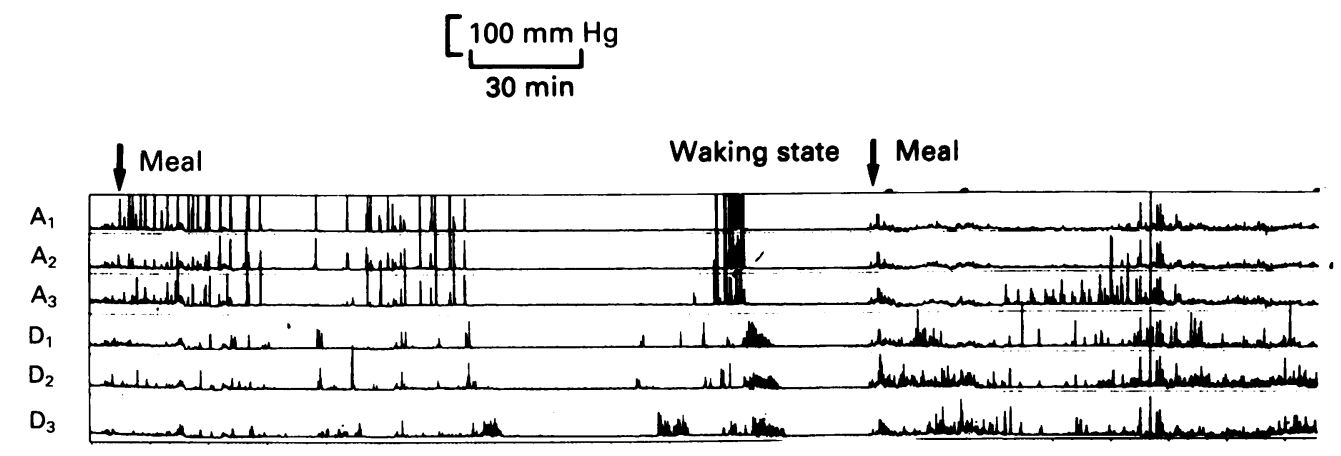

Sleep

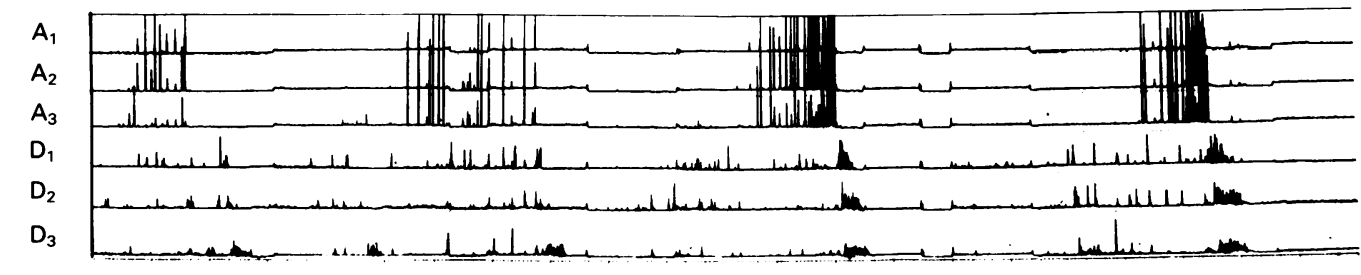

Figure 1: Prolonged ambulatory records of antral $\left(A_{1}-A_{3}\right)$ and duodenal $\left(D_{1}-D_{3}\right)$

motor activity.

(A) Representative record

from a healthy normal

volunteer. Shown is a

continuous tracing showing

an 11 hour long segment of

antroduodenal motor

activity. The upper trace

shows activity during

waking hours (starting at

$430 \mathrm{pm}$ ), the lower trace

activity during sleep. The

tracing was stopped at

$330 \mathrm{am}$. The subject had

one phase III migrating

motor complex during

waking hours and four

migrating motor complex

phase III during sleep;

(B) representative record

from a non-ulcer dyspepsia

patient. Shown is a

continuous 15.5 hour

segment, beginning at

$330 \mathrm{pm}$. The tracing was

stopped at 7 am. Only one

migrating motor complex

phase III was seen

45 minutes before

awakening. The dots

above the records shows

when the patient reported

abdominal pain;

(C) tracing from non-ulcer

dyspepsia +irritable bowel

syndrome patient. Shown is

a five hour continuous

segment of antroduodenal

motor activity, starting at

7 pm. The upper tracing

was obtained during

waking hours, the bottom

tracing during sleep. Dots

above the records show

when patient experienced

pain. Patient awoke twice

with pain; the second time

pain was followed by

retching (arrow). For

clarity, only one antral and

two duodenal recording sites

have been selected.

B
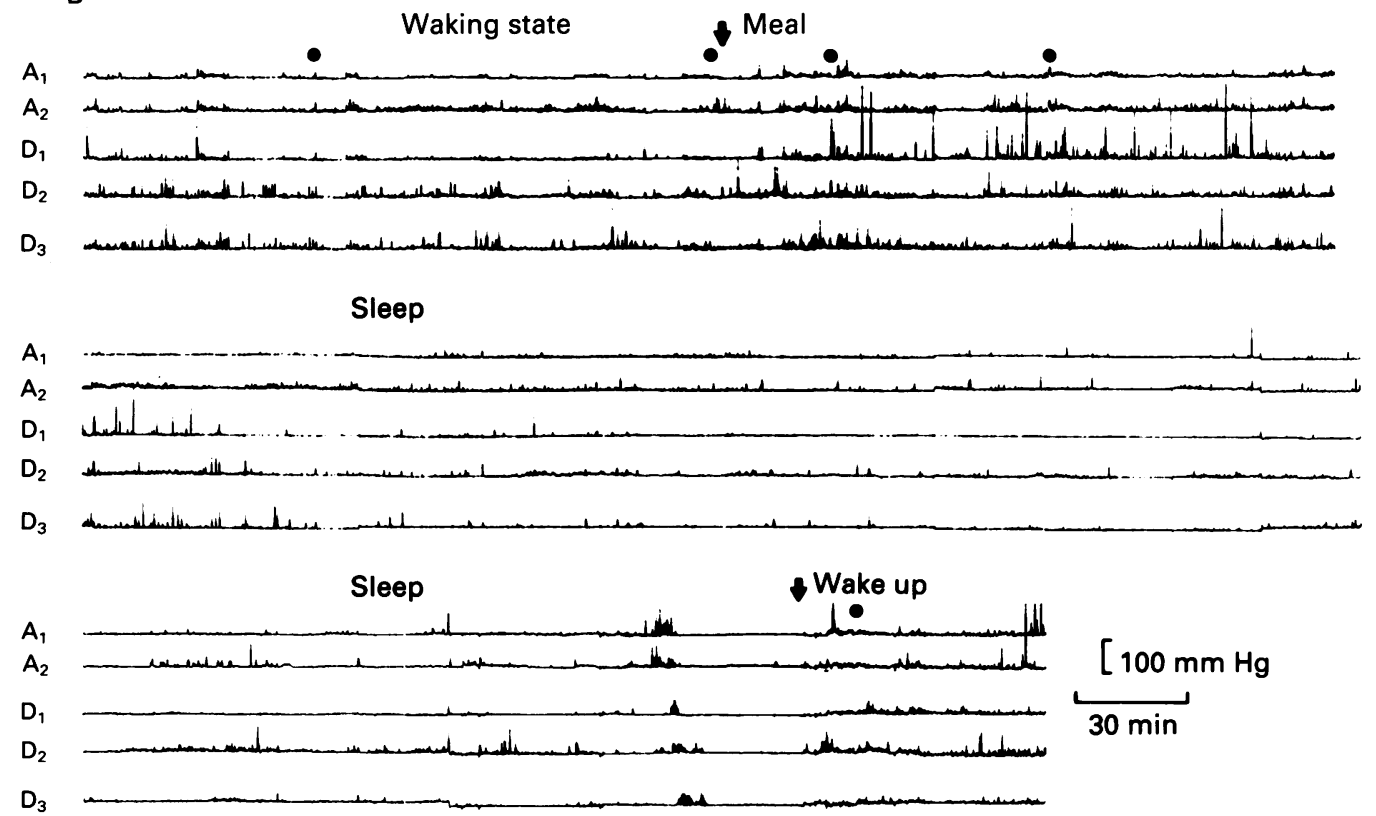

C
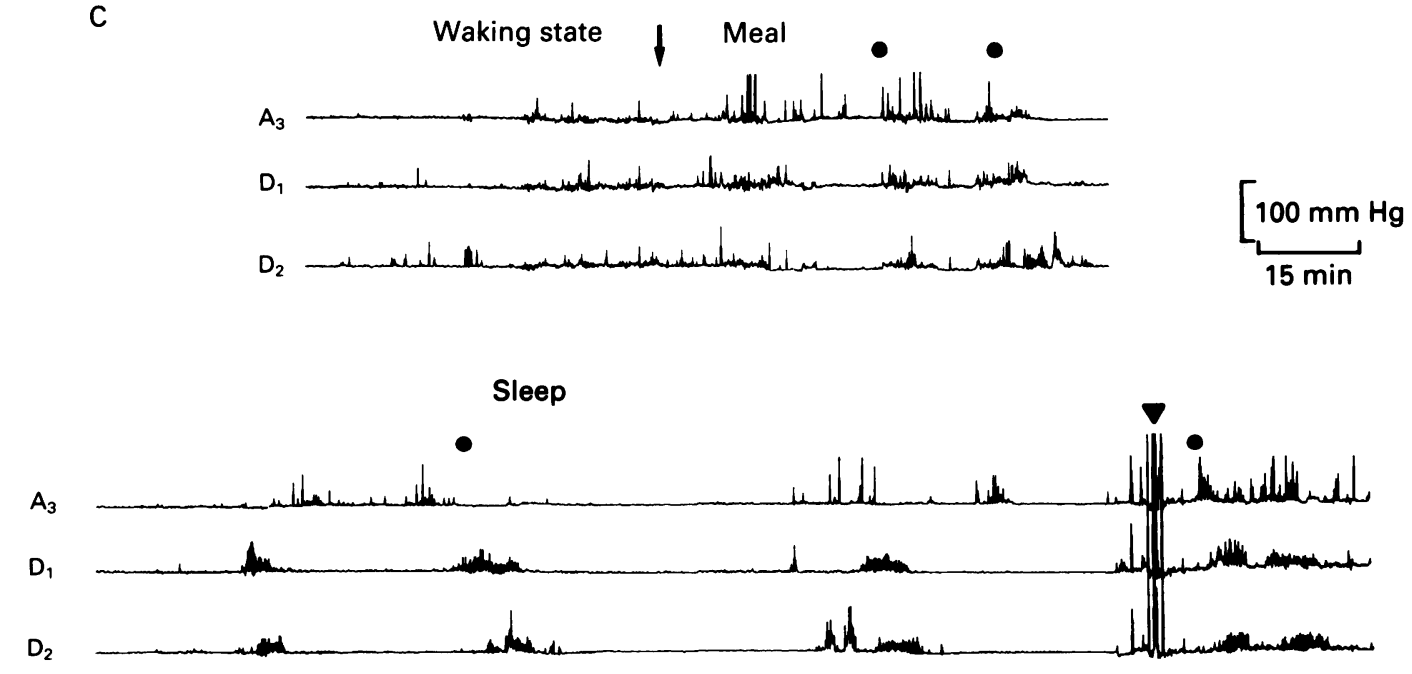
on the prevalence of poor sleep. Only one control subject reported difficulty falling asleep.

When subjects were asked to rate the discomfort and the quality of their sleep during the night of the manometric study, all normal subjects and all patients indicated that they slept worse than without the catheter. Patients experienced significantly more discomfort than controls. One of 10 normal controls and four of 11 patients reported difficulties falling asleep. Two controls and eight patients (three non-ulcer dyspepsia, five non-ulcer dyspepsia +irritable bowel syndrome) reported awakenings during the night. One patient (non-ulcer dyspepsia+irritable bowel syndrome) could not tolerate the catheter more than 14 hours because of discomfort.

\section{Circadian variation of motor activity}

The mean time spent in the nocturnal phase was $9 \cdot 2(1 \cdot 0) \mathrm{h}$ for controls and $8.9(1 \cdot 3) \mathrm{h}$ for patients.

Figure 1 shows representative tracings of diurnal and nocturnal motor activity from a control subject (A), from a non-ulcer dyspepsia patient (B), and from a nonulcer dyspepsia+irritable bowel syndrome patient (C). The most striking difference was a considerably decreased frequency of migrating motor complex phase III activity during sleep in non-ulcer dyspepsia patients. The comparative duration of migrating motor complex phases was calculated for each of the groups (Table III). The proportion of the various phases of motor activity did not differ significantly among the three groups during the diurnal recording period. In non-ulcer dyspepsia, 94 (1)\% were spent in phase II, 4 (1) $\%$ in phase I, and $2 \%$ in phase III. In contrast, when compared with the normal subjects there was a significant decrease in nocturnal phase I (5 (2) $v 78(6) \%$ ) and phase III activity ( $1 v 6(1) \%)$, and a significant increase in nocturnal phase II activity (94 (3) $v$ $15(2) \%)$ in the non-ulcer dyspepsia group. Thus, non-ulcer dyspepsia patients spent the same proportion (94\%) in phase II, regardless if they slept or were awake, while in normal subjects the proportion of phase II decreased by $84 \%$ during sleep. In contrast, in non-ulcer dyspepsia +irritable bowel syndrome patients, the nocturnal proportion spent in phase II was not different from normal controls.

TABLE IV Characteristics of the migrating motor complex (MMC) (mean (SEM))

\begin{tabular}{|c|c|c|c|}
\hline & $\begin{array}{l}\text { Controls } \\
(n=10)\end{array}$ & $\begin{array}{l}\text { NUD } \\
(n=5)\end{array}$ & $\begin{array}{l}\text { NUD+IBS } \\
(n=5)\end{array}$ \\
\hline $\begin{array}{l}\text { MMC/day (n) } \\
\text { MMC/night (n) }\end{array}$ & $\begin{array}{l}2 \cdot 5(0.4) \\
4.6(0.4)\end{array}$ & $\begin{array}{l}0.5(0.2)^{\star} \\
0.7(0.2)^{\star}\end{array}$ & $\begin{array}{l}1.5(0.2) \\
3.5(0.8)\end{array}$ \\
\hline $\begin{array}{l}\text { Diurnal phase 3: } \\
\text { Cycle length (min) } \\
\text { Duration (min) }\end{array}$ & $\begin{array}{l}238(53) \\
6.5(0 \cdot 5)\end{array}$ & $\begin{array}{l}\text { NA } \\
5 \cdot 8(0 \cdot 2)\end{array}$ & $\begin{array}{l}345 \\
4.8(0.4)\end{array}$ \\
\hline $\begin{array}{l}\text { Propagation velocity } \\
\text { (cm/min) }\end{array}$ & $6.9(0.7)$ & $2 \cdot 1(0 \cdot 3)^{\star}$ & $6.6(1.8)$ \\
\hline $\begin{array}{l}\text { Nocturnal phase 3: } \\
\text { Cycle length (min) } \\
\text { Duration (min) } \\
\text { Propagation velocity }\end{array}$ & $\begin{array}{l}120(14) \\
7 \cdot 2(0 \cdot 5)\end{array}$ & $\begin{array}{l}\text { NA } \\
7 \cdot 5(1 \cdot 5)\end{array}$ & $\begin{array}{l}111(15) \\
7 \cdot 3(0 \cdot 8)\end{array}$ \\
\hline $\begin{array}{l}\text { (cm/min) } \\
\text { Latency of phase } 3(\mathrm{~min})\end{array}$ & $\begin{array}{r}6.9(0.6) \\
40.4(0.8)\end{array}$ & $\begin{array}{r}7 \cdot 0(0 \cdot 6) \\
147(16)^{\star \star}\end{array}$ & $\begin{array}{r}5 \cdot 6(1 \cdot 8) \\
47 \cdot 7(1 \cdot 7)\end{array}$ \\
\hline
\end{tabular}

${ }^{\star} \mathrm{p}<0.05 v$ controls; ${ }^{\star \star} \mathrm{p}<0.005 v$ controls.
Characteristics of phase III activity

The characteristics of the migrating motor complex were studied in all subjects (Table IV). The number of migrating motor complexes during the diurnal and nocturnal period was significantly lower in the group with non-ulcer dyspepsia, whereas this value in non-ulcer dyspepsia + irritable bowel syndrome patients was not different from control subjects. In non-ulcer dyspepsia patients, the diurnal propagation velocity of phase III migrating motor complex $(2 \cdot 1(0 \cdot 3) \mathrm{cm} / \mathrm{min})$ was slower when compared with control subjects $(6.9(0.7) \mathrm{cm} / \mathrm{min})$ or when compared with non-ulcer dyspepsia +irritable bowel syndrome patients $(6.6(1.8) \mathrm{cm} / \mathrm{min})$. In contrast with previous reports, ${ }^{34}$ there was no difference in the propagation velocity between the diurnal or nocturnal periods in normal subjects or patients. The time from going to sleep (obtained from patient diary) until the first appearance of a migrating motor complex phase III was 40 (1) minutes in control subjects and 147 (16) in non-ulcer dyspepsia patients. There were no significant differences in the diurnal and nocturnal cycle length, phase III duration, or nocturnal propagation velocity among the three groups.

\section{Motor patterns}

Changes in motor patterns were noted in four non-ulcer dyspepsia + irritable bowel syndrome patients, but were not seen in any of the control subjects or the non-ulcer dyspepsia patients during the time of the study (Fig 2). Two patients had one such event $/ 24 \mathrm{~h}$ period with a duration of five and eight minutes respectively. Two patients had two such events $/ 24 \mathrm{~h}$ period, the duration ranging from 2-10 minutes. A change in duodenal motor activity was seen both during waking and sleeping hours. A change in motility was seen during fasting in the form of non-propagated clusters of phasic motor activity in the duodenum (A), prolonged high amplitude tonic contractions with superimposed phasic activity (B), and periodic propagated clusters of phasic activity (C). During the postprandial period, an increase in tonic motor activity with superimposed phasic contractions was seen (D). None of these motor events showed a temporal correlation with patients' reports of pain or discomfort.

\section{Abdominal pain}

Every patient reported pain or discomfort during the study. A total number of 55 pain episodes was reported by all patients (5.4 (1) $/ 24 \mathrm{~h} \mathrm{period/patient)} \mathrm{during} \mathrm{the} \mathrm{study}$ period. The frequency of pain episodes/patient was not different between non-ulcer dyspepsia $(5 \cdot 6(2) / 24 \mathrm{~h})$ and non-ulcer dyspepsia + irritable bowel syndrome $(5 \cdot 4(1) / 24 \mathrm{~h})$. In none of the patients was a temporal correlation of abdominal pain or discomfort correlated with changed motor events. As Figure 1(C) shows, however, one non-ulcer dyspepsia+irritable bowel syndrome patient reported consistently 
A
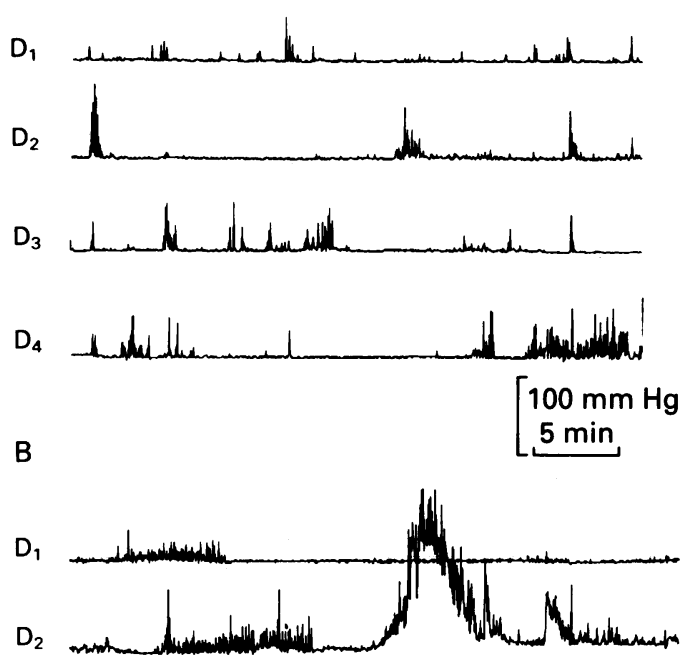

$\mathrm{D}_{3}$

Figure 2: Abnormal fasting $(A-C)$ and postprandial $(D)$ motor events not associated with abdominal pain. (A) Non-propagated clusters of phasic motor activity in the duodenum; (B) prolonged high amplitude tonic contraction with superimposed phasic contractions after a migrating motor complex phase III; (C) periodic clusters of contractile activity ('minute rhythm'); (D) prolonged increase in tonic motor activity with superimposed phasic contractions during postprandial period.

her typical pain during normal phase III of the migrating motor complex. This pain awakened the patient twice. In all other patients, pain was either associated with normal motor activity, or came before a change in motor activity (Fig 3 ).

\section{Discussion}

In this study we have shown that two thirds of patients seen at a tertiary referral centre with a complaint of abdominal discomfort or pain, or both thought to arise from the upper gastrointestinal tract complain of poor sleep. In addition, a change in rhythmicity of nocturnal

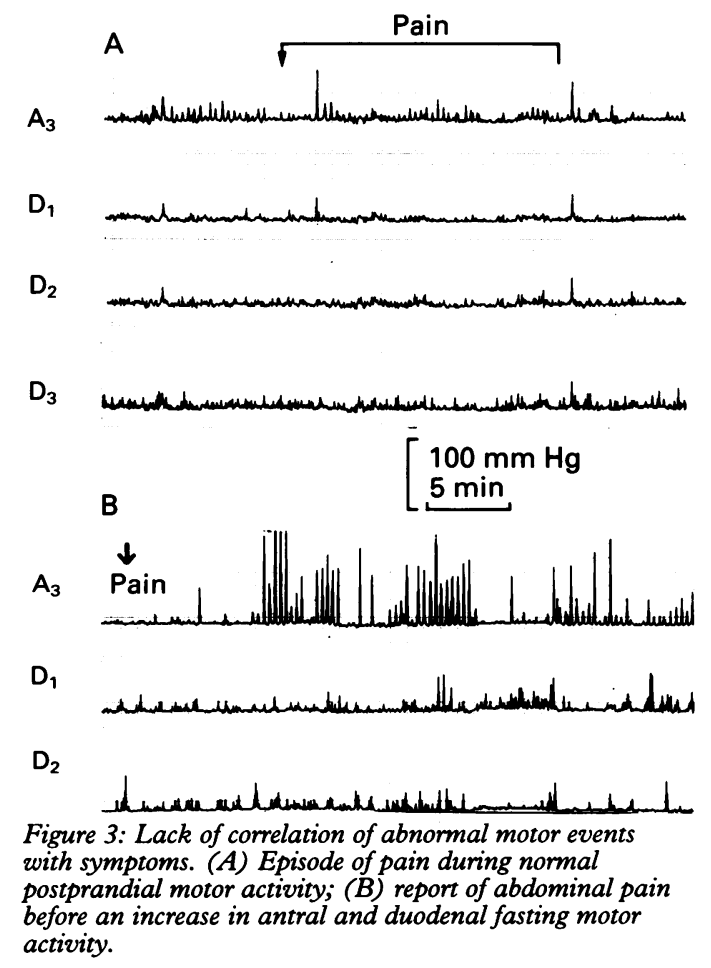

fasting motor activity was found in five of 10 non-ulcer dyspepsia patients.

Several human physiological functions seem to vary with a periodicity of 90 minutes. Such periodicity is seen in the form of the cyclic fasting gut motor activity, ${ }^{35}$ and in a change of REM and non-REM sleep. ${ }^{37}{ }^{38}$ Sleep has been divided into five stages based on differences in the predominant frequency distribution of the electroencephalogram. ${ }^{12}$ Stages 2 and 4 are generally combined and referred to as non-REM sleep. REM sleep sets in about 90 minutes after the onset of sleep, and REM and non-REM sleep alternate subsequently at about 90 minute intervals. Non-REM sleep is considered the quiescent period of sleep, during which autonomic activity decreases to its minimum. ${ }^{39}$

Early studies under non-ambulatory conditions showed a correlation between the periodicities of the REM-non-REM sleep cycle and fasting duodenal motor activity, ${ }^{40}$ while others could not confirm such a correlation. ${ }^{41}$ The correlation was taken as evidence for the existence of a basic neurological oscillatory mechanism that controlled the periodicity of both sleep and gut motility. When small bowel motility was measured during freely ambulatory conditions, the diurnal state was characterised by prolonged phase II activity (with comparatively little phase I and phase III activity), while in the nocturnal state phase II activity was virtually absent. ${ }^{34}$

Five of 10 patients studied were found to have a change in rhythmicity of nocturnal duodenal motor activity, primarily in the form of increased phase II activity. The possible role of female steroid hormones in the change of duodenal motor activity ${ }^{28} 42$ was not considered directly in this study. Several findings, however, make it unlikely that such hormonal influences were responsible for the current 
results. Firstly, non-ulcer dyspepsia and nonulcer dyspepsia+irritable bowel syndrome groups had a similar percentage of menstruating and non-menstruating women. Secondly, exacerbations of dyspeptic symptoms synchronous with the menstrual cycle were reported by few patients enrolled in this study. Thirdly, in previous studies using prolonged ambulatory monitoring of intestinal motor activity in humans, no difference between male and female subjects was reported. ${ }^{26}$ Fourthly, while female steroid hormones have been suggested to play a part in the regulation of gastric emptying and gastrointestinal transit, ${ }^{42}$ these effects have largely been explained by the negative inotropic effect of progesterone on gastrointestinal smooth muscle. ${ }^{43}$ In contrast, no consistent effect of female steroid hormones on fasting myoelectric activity has been found. ${ }^{28}$

Several indirect pieces of evidence suggest that phase II activity reflects increased CNS arousal. We and others ${ }^{34}$ have shown that phase II activity in normal subjects and in patients decreases soon after going to sleep and promptly increases upon awakening (Fig 1). Similarly, the electroencephalogram pattern associated with arousal, the alpha electroencephalogram typically disappears with onset of sleep and reappears during arousal from sleep. Disruption of gut brain communication - which mediates the effect of CNS arousal on gut function - by vagal cooling has been found to decrease the percentage of phase II activity in animals. ${ }^{44}$ If phase II activity reflects increased CNS arousal, it could be argued that the increase in nocturnal phase II activity seen in one half of patients is simply related to the increased discomfort (and therefore poor sleep) resulting from the test situation in these subjects. Even though all subjects slept worse during the test compared with their normal sleep, and normal control subjects did report less discomfort from the study when compared with patients, there was no difference in the reported discomfort between those patients who had increased phase II activity and those who did not. Furthermore, there was no correlation of the nocturnal motor pattern with the site at which the patient spent the night of the test. Thus sleeping in an unfamiliar environment (hotel, clinical study centre) did not make it more probable for increased phase II activity to occur. Similarly, none of the control subjects who slept in a hospital bed showed the nocturnal motor pattern. Another variable that could have resulted in prolonged phase II like activity is a difference in gastric emptying rates. Even though mildly delayed gastric emptying was noted in five patients, it could hardly have accounted for the absence of phase III activity during the entire nocturnal period. In addition, delayed gastric emptying was present in two non-ulcer dyspepsia + irritable bowel syndrome patients who had normal nocturnal motor patterns.

A variety of studies show a relation between a change in sleep physiology with symptoms of chronic unrefreshing sleep, musculoskeletal pain/fibromyalgia syndrome, dysphoric mood, and irritable bowel syndrome. $\begin{array}{lllllll}15 & 17 & 18 & 20 & 21 & 45\end{array}$ The prolonged disruption of stage 4 or slow wave (deep) sleep in healthy subjects results in the emergence of gastrointestinal symptoms such as nausea, abdominal cramping, and diarrhoea, and somatic symptoms that include musculoskeletal complaints and fatigue. ${ }^{46}$ It is felt that sleep deprivation may lead to a daytime hyperalgesic state, with increased sensitivity to several normal bodily functions. This hypothesis is supported by recent evidence showing that sleep deprivation can result in a lowering of rectal sensory thresholds to balloon distension ( $N$ Read, personal communication).

Gastrointestinal symptoms that awaken the patient from sleep have generally been considered to be of organic origin. ${ }^{12}$ Our study confirms previous reports, however, that subjective reports of poor sleep are common in patients with functional bowel symptoms. ${ }^{13} 14$ Furthermore, our findings suggest a possible correlation between poor sleep, increased nocturnal phase II motor activity, and the presence of chronic visceral pain syndromes.

Possible mechanisms to explain the increase in nocturnal phase II motor activity seen in this study include the effect of chronic distress on sleep, or the stimulation of sensitised visceral afferents by mechanical or chemical events, or both related to the presence of the manometry tube. Such stimulation could result in increased afferent input to brainstem areas concerned with the regulation of the wakesleep cycle. ${ }^{47} 48$ For example, appearance of the 'alpha electroencephalogram abnormality' has been reported to occur in response to inflation of a balloon catheter in the oesophagus during sleep. ${ }^{49}$ Oesophageal chemoreceptor stimulation associated with spontaneous relaxation of the lower oesophageal sphincter and resulting in gastro-oesophageal reflux, has been found to be associated with arousal from sleep. ${ }^{50}$ Gastro-oesophageal reflux may be facilitated by the manometry tube.

Our findings of increased nocturnal phase II motor activity in patients who only complain of symptoms thought to arise from the upper gut (non-ulcer dyspepsia), differ from those who also complained of symptoms thought to arise from the lower gut (non-ulcer dyspepsia +irritable bowel syndrome), even though both group of patients complained of poor sleep. It has previously been reported that patients with a diagnosis of irritable bowel syndrome have similar nocturnal motor patterns as normal controls. ${ }^{44}$ Similarly, a recent study of irritable bowel syndrome patients and healthy controls by Kumar et al ${ }^{27}$ found no differences between the two groups in the nocturnal intestinal motility pattern. The number of migrating motor complexes during sleep was within normal limits, and there was very little nocturnal phase II activity in either group. The proportion of time spent in REM was significantly increased in irritable bowel syndrome patients compared with controls. It is conceivable that lowered mechanoreceptor (or chemoreceptor, or both) thresholds in the 
oesophagus or stomach, or both (a finding common in non-ulcer dyspepsia patients) results in a greater likelihood of sleep disruption by normal physiological events, or in response to the manometry tube. Even though we did not characterise gastric sensory thresholds in our patients, the fact that normal or abnormal duodenal motor events did not correlate with discomfort except in one patient with non-ulcer dyspepsia+irritable bowel syndrome, while similar small intestinal motor events are associated with abdominal discomfort in irritable bowel syndrome patients ${ }^{26}$ suggests possible regional differences in visceral hypersensitivity.

As shown in this study and previous reports, ${ }^{13} 1420$ sleep disturbances including the 'alpha abnormality' are not restricted to non-ulcer dyspepsia patients but are common in patients with irritable bowel syndrome symptoms as well. In a study of 123 patients with fibromyalgia syndrome (thought to exhibit the 'alpha abnormality'), discomfort or pain, or both thought to arise from the lower gastrointestinal tract, and consistent with a diagnosis of irritable bowel syndrome were found in $54 \%$ of patients, while complaints of a change in bowel habits in these patients were not different from control subjects without fibromyalgia syndrome. ${ }^{20}$ Nausea was found in $21 \%$.

Our findings indirectly support an association between changed nocturnal duodenal motor activity and a changed sleep pattern in some non-ulcer dyspepsia patients. Currently available data ${ }^{17} \quad 20 \quad 27 \quad 40$ suggest that based on specific sleep abnormalities, there may be distinct subgroups of patients with functional bowel disorders. One could speculate that those patients who exhibit the 'alpha abnormality' may show increased responsiveness to treatment with low dose tricyclic antidepressants, similar to fibromyalgia syndrome patients. Formal polysomnographic studies in these patients and correlation of sleep patterns with motor and sensory function of the upper gut will be necessary to further test this hypothesis.

This study was supported in parts by NIH grant DK 40919 and by funds from the Veterans' Administration.

1 Talley NJ, Phillips SF. Non-ulcer dyspepsia: potential causes and pathophysiology. Ann Intern Med 1988; 108: 865-79.

2 Barbara L, Camilleri M, Corinaldesi R, Crean GP Heading RC, Johnson AG, et al. Definition and investigation of dyspepsia. Consensus of an international ad hoc tion of dyspepsia. Consensus of an internation
working party. Dig Dis Sci 1989; 34: 1272-6.

3 Talley NJ, Zinsmeister AR, Schleck CD, Melton LJ. Dyspepsia and dyspepsia subgroups: a population-based study. Gastroenterology 1992; 102: 1259-68.

4 Kerlin P. Postprandial antral hypomotility in patients with idiopathic nausea and vomiting. Gut $1989 ; 30: 54-9$. Malagelada J-R, Stanghellini V. Manometric evaluation of functional upper gut symptoms. Gastroenterology 1985 88: 1223-31

6 Waldron B, Cullen PT, Kumar R, Smith D, Jankowski J, Hopwood D, et al. Evidence for hypomotility in non-ulcer dyspepsia: a prospective multifactorial study. Gut 1991; 32: $246-51$.

7 Talley NJ, Shuter B, McCrudden G, Jones M, Hoschl R Piper DW. Lack of association between gastric emptying of solids and symptoms in nonulcer dyspepsia. of solids and symptoms in no

8 Camilleri M, Neri M. Motility disorders and stress. Dig Dis Sci 1989; 34: 1777-86.

9 Talley NJ. Spectrum of chronic dyspepsia in the presence of the irritable bowel syndrome. Scand $\mathcal{f}$ Gastroenterol 1991; 182: 7-10.
10 Mearin F, Cucala M, Azpiroz F, Malagelada J-R. The origin of symptoms on the brain-gut axis in functional dyspepsia. Gastroenterology 1991; 101: 999-1006.

11 Coffin B, Azpiroz F, Malagelada J-R. Selective gastric hypersensitivity and reflex hyporeactivity in functional hypersensitivity and reflex hyporeactivity

12 Orr WC, Robinson MG. The sleeping gut. Med Clin North Am 1981; 65/6: 1359-75.

13 Whorwell PJ, McCallum M, Creed FH. Non-colonic features of irritable bowel syndrome. Gut 1986; 27 37-40.

14 Maxton DG, Morris J, Whorwell PJ. More accurate diagnosis of irritable bowel syndrome by the use of 'noncolonic' symptomatology. Gut 1991; 32: 784-6.

15 Moldofsky $\mathrm{H}$. The relevance of sleep to chronic pain. Rheum Dis Clin North Am 1989; 15: 91-103.

16 Boissevain $\mathrm{MD}$, McCain GA. Toward an integrated understanding of fibromyalgia syndrome. I. Medical and pathophysiological aspects. Pain 1991; 45: 227-38.

17 Moldofsky H, Franklin LA. Disordered sleep, pain, fatigue, and gastrointestinal symptoms in fibromyalgia, chronic fatigue and irritable bowel syndromes. In: Mayer chronic fatigue and irritable bowel syndromes. In: Mayer abdominal pain. New York: Elsevier, 1993: 249-56.

18 Moldofsky $\mathrm{H}$. The contribution of sleep - wake physiology to fibromyalgia. In: Fricton JR, Awad EA, eds. Advances in pain research. Vol 17. New York: Raven, 1990: 227-40.

19 Veale D, Kavanach G, Fielding JF, Fitzgerald O. Primary fibromyalgia and irritable bowel syndrome: differen expressions of a common pathogenetic process. Brf Rheumatol 1991; 30: 220-2.

20 Triadafilopoulos G, Simms RW, Goldenberg DL. Bowel dysfunction in fibromyalgia syndrome. Dig Dis Sci 1991; 36: $59-64$

21 Yunus M, Masi AT, Calabro J, Miller KA, Feigenbaum SL. Primary fibromyalgia (fibrositis): clinical study of 50 patients with matched nor

22 McCain GA. Management of the fibromyalgia syndrome. In: Fricton JR, Awad EA, eds. Advances in pain research. Vol 17. New York: Raven, 1990: 289-304.

23 Klein KB. Controlled treatment trials in the irritable bowel syndrome: a critique. Gastroenterology 1988; 95: 232-41

24 Max MB, Culnane M, Schafer SC, Gracely RH, Walther DJ, Smoller B, et al. Amitryptiline relieves diabetic neuropathy pain in patients with normal or depressed mood. Neurology 1987; 37: 589-96.

25 Feinmann C. Pain relief by antidepressants: possible modes of action. Pain 1985; 23: 18 .

26 Kellow JE, Gill RC, Wingate DL. Prolonged ambulant recordings of small bowel motility demonstrate abnormalities in the irritable bowel syndrome. Gastroenterology 1990; 98: 1208-18.

27 Kumar D, Thompson PD, Wingate DL, VesselinovaJenkins CK, Libby G. Abnormal REM sleep in the irritable bowel syndrome. Gastroenterology 1992; 103: 12-7.

28 Scott LD, Lester R, Van Thiel DH, Wald A. Pregnancyrelated changes in small intestinal myoelectric activity in the rat. Gastroenterology 1983; 84: 301-5.

29 Talley NJ, Phillips SF, Melton III J, Wilten C, Zinsmeister AR. A patient questionnaire to identify bowel disease. Ann Intern Med 1989; 111: 671-4.

30 Talley NJ, Phillips SF, Wiltgen CM, Zinsmeister AR Melton III LJ. Assessment of functional gastrointestinal disease: the bowel disease questionnaire. Mayo Clin Proc 1990; 65: 1456-79.

31 Drossman DA, Thompson GW, Talley NJ, Funch-Jensen $P$, Janssens J, Whitehead WE. Identification of subgroups of functional gastrointestinal disorders. Gastroenterology International 1990; 3: 159-72.

32 Manning AP, Thompson WH, Heaton KW. Towards positive diagnosis of the irritable bowel. BMF 1978; 2: 653-4.

33 Mayer EA, Sytnik B, Reddy SN, Van Deventer GM, Tache $\mathrm{Y}$. Corticotropin releasing factor (CRF) increases post-prandial duodenal motor activity in humans. fournal of Gastrointestinal Motility 1992; 4: 53-60.

34 Kellow JE, Gill RC, Wingate DL. Prolonged ambulant recordings of small bowel motility demonstrate abnorrecordings of small bowel motility demonstrate abnormalities in the irrita

35 Kellow JE, Borody TJ, Phillips SF, Tucker RL, Haddad AC. Human interdigestive motility: variations in pattern from oesophagus to colon. Gastroenterology 1986; 91 386-95.

36 Kellow JE, Phillips SF. Altered small bowel motility in irritable bowel syndrome is correlated with symptoms. Gastroenterology 1987; 92: 1885-93.

37 Hartmann E. The 90-minute sleep-dream cycle. Arch Gen Psychiatry 1968; 18: 280-6.

38 Kripke DF. Ultradian rhythms in sleep and wakefulness. In: Weitzman ED, ed. Advances in sleep research. Vol 1 . Jamaica, NY: Spectrum, 1974: 305-25.

39 Orem J, Barnes CD, eds. Research topics in physiology. Physiology in sleep. New York: Academic Press, 1980: Physiology

40 Finch PM, Ingram DM, Henstridge JD, Catchpole BN. Relationship of fasting gastroduodenal motility to the Relationship of fasting gastroduodenal motility
sleep cycle. Gastroenterology 1982; 83: 605-12.

41 Talley NJ, Camilleri M, Orkin BA, Kramlinger KG. Effect of cyclical unipolar depression on upper gastrointestinal motility and sleep. Gastroenterology 1989; 97: 775-7. 
42 Everson GT. Gastrointestinal motility in pregnancy. Gastroenterol Clin North Am 1992; 21 (4): 751-76.

43 Bruce LA, Behsudi FM. Progesterone effects on three regional gastrointestinal tissues. Life Sci 1979; 25: regional

44 Chung SA, Diamant NE. Small intestinal motility in fasted and fed postprandial states: effect of transient vagosympathetic blockade. Am f Physiol 1987; 252: G301-8.

45 McCain GA, Scudds RA. The concept of fibromyalgia (fibrositis): clinical value, relation and significance to other chronic musculoskeletal pain syndromes. Pain 1988; 33: 273-87.
46 Moldofsky H, Scarisbrick PS. Induction of neurasthenic musculoskeletal pain syndrome by selective sleep stage deprivation. Psychosom Med 1976; 38: 35-44.

47 Klemm WR, Vertes RP. Brainstem. Mechanisms of behavior. New York: Wiley, 1990.

48 Kelly DD. Sleep and dreaming. In: Kandel ER, Schwartz $\mathrm{JH}$, eds. Principles of neural science. New York: Elsevier, 1985: 648-70.

49 Chediak AK, Demirozu MC, Nay KN. Alpha EEG sleep produced by balloon catheterization of the esophagus. Sleep 1990; 13: 369-70.

50 Dent J, Dodd WJ, Friedman RH. Mechanism of gastroesophageal reflux in recumbent asymptomatic human subjects. $\mathcal{F}$ Clin Invest 1980; 65: 256-7. 